\title{
Atypical bartonellosis in children: What do we know?
}

\author{
Ana P Lemos ${ }^{1},{ }^{1}$ Rui Domingues, ${ }^{1}$ Catarina Gouveia, ${ }^{1}$ Rita de Sousa ${ }^{2}$ and Maria J Brito ${ }^{1}$ \\ ${ }^{1}$ Department of Infectious Diseases, Hospital Dona Estefânia and ${ }^{2}$ Centre of Study of Vectors and Infectious Diseases, National Institute of Health Dr. \\ Ricardo Jorge, Lisbon, Portugal
}

\begin{abstract}
Aim: To characterise Bartonella infections in a paediatric population requiring hospital admission and review its treatment.
Methods: Longitudinal observational retrospective data analysis of children and adolescents admitted with Bartonella infection at a paediatric tertiary hospital from 2010 to 2019.

Results: We identified 16 cases of bartonellosis, with a mean age of $8.0 \pm 4.5$ years old, no sex predominance and 14 had contact with cats. Most of the cases occurred in fall and winter. Clinical presentations included osteomyelitis/arthritis $(n=9)$, hepatosplenic disease $(n=2)$, lymphadenitis $(n=2)$, neuroretinitis $(n=2)$ and encephalitis $(n=1)$. Clinical diagnosis was confirmed by serology $(n=16)$ and Bartonella DNA detection in patient's lymph nodes/hepatic lesion $(n=3)$. Therapeutic approach varied according to the clinical presentation: azithromycin in lymphadenitis, rifampicin plus ciprofloxacin in hepatosplenic disease, rifampicin and doxycycline in neuroretinitis, ceftriaxone in encephalitis and azithromycin, cotrimoxazole or rifampicin plus azithromycin, cefuroxime, ciprofloxacin or doxycycline in osteomyelitis/arthritis. Immunodeficiency was excluded in seven patients. Seven patients' cats were screened by veterinarians and treated when infected $(n=5)$.

Conclusions: In these clinical presentations, where other infections may be involved, a high index of suspicion is necessary, with emphasis on the epidemiological context. The association of systemic forms with immunodeficiency did not occur in our study. The lack of recommendations for treatment of atypical infection makes the approach of these cases a challenge. Randomised control studies are essential to define the best approach in each case.
\end{abstract}

Key words: Bartonella infection; encephalitis; fever of unknown origin; lymphadenitis; osteomyelitis; retinitis.

What is already known on this topic

1 Bartonella infection usually has a benign course, but systemic involvement may occur in a number of patients.

2 Atypical manifestations are more common in immunocompromised patients, but can occur in immunocompetent patients.

3 Antibiotic therapy in all patients with bartonellosis is still controversial.

\section{What this paper adds}

1 Atypical manifestations of Bartonella infection, particularly bone and joint infection, were more frequent than previously reported.

2 Bartonella infection should be included in the differential diagnosis of the previously described clinical entities and a high index of suspicion, with emphasis on the epidemiological context, is required.

3 Even though multiple antibiotic regimens were used, all patients fully recovered, so there is no mandatory specific treatment for atypical infection.
The most prevalent bartonellosis in children is cat scratch disease (CSD), a benign zoonotic infectious disease, typically caused by Bartonella henselae and sporadically by Bartonella clarridgeiae. Cats are the natural reservoir of these bacteria and may infect humans through scratches, bites or fleas. ${ }^{1}$ Dogs have also been implicated in $5 \%$ of cases. ${ }^{2,3}$ Epidemiological investigations show that contact history with cats is present in around $90 \%$ and the antecedent of scratch or bites in $60 \%$ of the patients. ${ }^{4}$ Most cases occur in children and adolescents, usually male, and have been consistently reported to peak in autumn and winter months. ${ }^{5}$ The

Correspondence: Dr Ana P Lemos, Department of Infectious Diseases, Hospital Dona Estefânia, Rua Jacinta Marto 1169-045, Lisbon, Portugal. Fax: +351 213126988; Email: ana1645@gmail.com

Conflict of interest: None declared.

Accepted for publication 17 November 2020 most frequent presentation of CSD is a self-limited regional lymphadenopathy, which can be associated with malaise, fever, anorexia, headache, rash or arthralgia. In few cases, mainly in immunocompromised patients, ${ }^{6}$ a wide clinical spectrum and systemic involvement including cutaneous, hepatosplenic, ophthalmological, neurological and musculoskeletal manifestations can occur and might result in major illness.

The diagnosis of these atypical presentations is usually difficult, and a high index of suspicion is mandatory. The hepatosplenic disease is often associated with prolonged fever and microabscesses in the liver and/or spleen. ${ }^{7}$ Ophthalmological manifestations include the Parinaud's oculoglandular syndrome, that associates fever, regional lymphadenopathy and follicular conjunctivitis. In few cases, other manifestations reported are uveitis, neuroretinitis, inflammatory disc oedema, retinal vessel occlusions and vasculitis. ${ }^{8}$ Nervous system involvement most commonly presents as 
encephalopathy, but meningomyeloradiculopathy, nerve palsies, Guillain-Barre Syndrome, acute hemiplegia, transverse myelitis and cerebral arteritis have been reported. ${ }^{9}$ As for the musculoskeletal involvement, osteomyelitis and arthritis are the most common presentations and are usually concomitant with hepatosplenic disease.

The treatment of these atypical presentations in immunocompetent patients is controversial. While some cases can resolve spontaneously without treatment, antibiotic therapy may prevent serious complications and shorten the duration of symptoms. ${ }^{10}$ Standardised treatment recommendations for atypical bartonellosis do not exist, and thus, antibiotic recommendations differ for each clinical presentation. ${ }^{11}$

The aim of this study was to characterise Bartonella infections in a paediatric population requiring hospital admission and review its treatment in atypical presentations.

\section{Methods}

Observational retrospective data analysis of children and adolescents $(<18$ years) admitted with bartonellosis at a paediatric tertiary hospital from January 2010 to December 2019. Confirmed case was based on epidemiological history, clinical and laboratory confirmation by serology and/or molecular detection of Bartonella DNA in clinical specimens. Additionally, in some cases immunohistochemistry (Warthin-Starry silver stain) was performed in biopsy specimens.

Serological diagnosis of Bartonella infection was confirmed by indirect immunofluorescence assay (IFA). We used commercial slides (Bartonella IFA IgG and IgM; Focus Diagnostics Technologies, Cypress, California, USA) to determine antibodies against Bartonella spp. Confirmed case was defined when acute serum contained IgM antibodies at a titre of $\geq 32$ and IgG titre of $\geq 128$ or there was a four-fold increase in IgG titre between acute and convalescent sera. Convalescent serum was collected at least 15 days after the acute serum. DNA was extracted from clinical specimens (blood/lymph node/hepatic lesions/aspirate of purulent exudate) using the automated MagCore nucleic acid extractor instrument (RBCBioscience). Bartonella DNA was detected by nested-polymerase chain reaction (PCR) of a fragment of riboflavin synthase chain (RibC) gene as previously described. ${ }^{12}$ Positive amplicons were sequenced and analysed by comparison with the sequences available in the NCBI (GenBank) nucleotide database (http://blast.ncbi.nlm.nih.gov/Blast).

Ethical committee approval was obtained and the study was conducted in accordance with ethical standards and the Helsinki Declaration.

\section{Results}

We identified 16 patients with bartonellosis, 14 with atypical presentation $(88 \%)$. Mean age \pm standard deviation was $8.0 \pm 4.5$ years old (range $1-17$ years), without sex predominance. Cat exposure was reported in 14 patients $(88 \%)$ and five had recent scratch or bite $(35 \%)$. We also confirmed that five of the patient's cats tested positive by PCR for Bartonella spp. Seven patients had contact with dogs $(44 \%)$. Most cases occurred in summer $(n=7)$ and fall $(n=8)$. Symptoms started on an average of $11 \pm 7.3$ days before the first evaluation and the mean duration between the onset of symptoms and diagnosis was $44 \pm 14.7$ days.

Fever of unknown origin was reported in eight patients $(50 \%)$ and regional lymphadenopathy in $11(69 \%)$ : submandibular $(n=4)$, inguinal $(n=3)$, axillary $(n=2)$, cervical $(n=2)$, epitrochlear $(n=1)$ and supraclavicular $(n=1)$. Scratch marks or the classic CSD papule were not reported.

Thirteen patients had a normal white blood cell count and differential with a mean white blood cell count of $10.2 \pm 2.3 \times 10^{9}$ cells/L. Erythrocyte sedimentation rate and C-reactive protein (CRP) were elevated in 15 patients with a mean of $54.4 \pm 22.2 \mathrm{~mm} / \mathrm{h}$ and $76.5 \pm 55.2 \mathrm{mg} / \mathrm{L}$, respectively. Two patients had normochromic normocytic anaemia and blood cultures were negative in all patients. Human immunodeficiency virus infection and interleukin deficits were excluded in seven patients.

Seroconversion of IgG antibodies against Bartonella spp. in sera was confirmed in all cases (Table 1). Except for patient 6, all patients were positive for IgG in the first sample. IgM was positive in five cases $(31 \%)$. The period of time between consecutive tested samples from each patient varied between 10 days and 11 months and therefore in some patients the titers increased in relation to the previous sample and in others we observed a decrease in particular in patients with intervals greater than 3 months.

Besides the confirmed seroconversion, two children (Table 1) had confirmed diagnosis by PCR: patient 3 in lymph node and hepatic lesion and patient 6 in lymph node. Blood PCR was negative in seven tested patients, all of them under antibiotics. Histopathologic examination of the affected lymph nodes was performed in four patients $(25 \%)$, and revealed granulomatous adenitis in two, as well as microabscesses and follicular hyperplasia in one. Warthin-Starry silver impregnation stain was negative in all cases.

The clinicopathologic subtypes of the disease, shown in Table 2, included lymphadenitis $(n=2)$, hepatosplenic disease $(n=2)$, neuroretinitis $(n=2)$, encephalitis $(n=1)$ and osteomyelitis/arthritis $(n=9)$.

Among the two patients with lymphadenitis the longer duration of symptoms justified hospital admission. Both presented with inguinal lymphadenopathy, lasting more than 3 weeks, and one required lymph node biopsy, to exclude alternative diagnosis. Antibiotic therapy with azithromycin lead to clinical improvement in both cases.

Hepatosplenic disease presented as prolonged fever of more than 3 weeks, submandibular/axillary lymphadenopathy and microabscesses in the liver and/or spleen. None had organomegalies on physical examination, but one child had splenomegaly on the abdominal ultrasound. The higher levels of CRP were associated with this atypical presentation, with a maximum of $206.4 \mathrm{mg} / \mathrm{L}$. Lymph node and hepatic lesion biopsy were performed in one child, and confirmed the presence of Bartonella's DNA in the specimen and the presence of granulomatous adenitis in the histopathological exam. Both patients were treated with rifampicin and ciprofloxacin and clinically improved.

Neuroretinitis presented as prolonged fever and unilateral vision loss, in one case associated with intracranial hypertension (opening pressure of $28 \mathrm{~cm} \mathrm{H}_{2} \mathrm{O}$ ). The diagnosis of retinitis was confirmed by an ophthalmologist and both patients were treated 
Table 1

IFA titres of all patients' samples

\begin{tabular}{|c|c|c|c|c|c|c|c|c|}
\hline \multirow{3}{*}{ Patient no. } & \multicolumn{8}{|c|}{ IFA titres of patients samples } \\
\hline & \multicolumn{2}{|c|}{ First sample } & \multicolumn{2}{|c|}{ Second sample } & \multicolumn{2}{|c|}{ Third sample } & \multicolumn{2}{|c|}{ Forth sample } \\
\hline & $\lg M$ & IgG & $\lg M$ & IgG & $\lg M$ & $\lg G$ & $\lg M$ & $\lg G$ \\
\hline 1 & - & 512 & - & 256 & - & 128 & NA & NA \\
\hline 2 & - & 256 & NA & NA & NA & NA & NA & NA \\
\hline $3 \dagger$ & 64 & 8192 & - & 4096 & - & 2048 & - & 1024 \\
\hline 4 & 32 & 2048 & - & 2048 & - & 512 & - & 256 \\
\hline 5 & - & 64 & - & 512 & NA & NA & NA & NA \\
\hline $6 \dagger$ & - & - & - & 1024 & - & 512 & NA & NA \\
\hline 7 & - & 8192 & 512 & 16384 & NA & NA & NA & NA \\
\hline 8 & $\geq 32$ & 2048 & 128 & 4096 & NA & NA & NA & NA \\
\hline 9 & - & 512 & - & 2048 & - & 512 & NA & NA \\
\hline 10 & - & 128 & - & 256 & - & 256 & NA & NA \\
\hline 11 & $\geq 32$ & 4096 & NA & NA & NA & NA & NA & NA \\
\hline 12 & - & 64 & - & 1024 & NA & NA & NA & NA \\
\hline 13 & - & 1024 & - & 4096 & NA & NA & NA & NA \\
\hline 14 & - & 2048 & - & 512 & NA & NA & NA & NA \\
\hline 15 & $\geq 32$ & 2048 & - & 512 & NA & NA & NA & NA \\
\hline 16 & - & 1024 & - & 512 & $N A$ & NA & $N A$ & NA \\
\hline
\end{tabular}

$\dagger$ Polymerase chain reaction positive patients. IgG titers were positive if $\geq 128$ and IgM titers were positive if $\geq 32$. NA, not available; - , negative for IgM or IgG.

with corticosteroids, rifampicin and doxycycline with full recovery.

Neurologic involvement presented as encephalopathy in a child with fever, focal seizures and hemiparesis. Lumbar puncture revealed pleocytosis (2l cells/uL mononuclear cells) and proteinoraquia $(80 \mathrm{mg} / \mathrm{dL})$. CSF PCR for Bartonella spp. was negative. Empiric treatment with ceftriaxone was initiated and clinical resolution was achieved before the etiologic diagnosis.

As for the musculoskeletal involvement, all children had pain, one claudication, eight had fever, four of unknown origin, six had lymphadenopathy and one torticollis. The affected areas in these osteoarticular presentations were the vertebral spine $(n=5)$, the sacroiliac joint $(n=3)$, the sternoclavicular joint $(n=1)$ and the tibiotarsal joint $(n=1)$. One patient had multifocal involvement (11\%). Two children had hepatosplenic microabscesses $(22 \%)$. PCR of a lymph node biopsy performed in one child revealed the presence of Bartonella' DNA and the histopathological exam confirmed the presence of granulomatous adenitis. All patients were treated with antibiotics, ranging from azithromycin, cotrimoxazole or rifampicin plus azithromycin, cefuroxime, ciprofloxacin or doxycycline.

\section{Discussion}

Atypical manifestations of bartonellosis among immunocompetent patients are rare $(5-14 \%) \cdot .^{1,13}$ Thereby, based on the clinical features and a compatible epidemiological context, CSD diagnosis should be evoked and confirmed with accurate diagnostic exams.

In our study, cat exposure had a similar value to the expected $87-99 \%$, but a scratch and/or bite were only documented in five cases $(35 \%)$, opposing the previously reported $50 \%{ }^{4}$ As our sample included only paediatric patients, opposing to Carithers's study, ${ }^{13}$ the forgetfulness of an encounter with a cat, that occurred more than a week before, is very likely in this group of age and can explain our outcomes. The contact with dogs may also have been relevant. As expected, clinical evaluation was made on average after 11 days of symptoms and the mean duration between the onset of symptoms and the diagnosis was 44 days. At this stage of the evolution of the disease most patients already have antibodies, and it is more difficult to detect the bacteria in the blood. In some cases, however, the bacteria (DNA) is still detectable in the lymph nodes, reinforcing that, for many days after the onset of disease, the lymph node, hepatic lesions or purulent exudate are better specimens to test for PCR, since bacteria are more difficult to be removed compared to the blood circulation. Laboratory findings, are known to be nonspecific; infection may result in leukocytosis and normal or elevated ESR and CRP levels and mild anaemia and elevated levels of the liver enzymes can also be found in some cases with systemic involvement. ${ }^{9}$

This wide clinical spectrum of disease in immunocompetent patients, makes it essential to exclude other serious conditions that can mimic CSD. Thus, the diagnostic techniques tests have been applied for the detection of Bartonella spp. In the present study, the diagnosis was confirmed in all cases by the seroconversion of antibodies against Bartonella in IFA using Bartonella henselae and Bartonella quintana as slides antigen. However, since serological cross-reactions may occur only molecular tests using specific probes by real-time PCR or conventional PCR and sequencing can confirm Bartonella species involved in the infection. Due to the high mean duration between the onset of symptoms and the diagnosis, IgM was only positive in $31 \%$ of the cases, indicating the delay on the diagnosis. As expected, due to its invasive nature, histopathologic examination of the affected lymph nodes was only 
Table 2 Socio-demographic, epidemiological, clinical, diagnostic and therapeutic characteristics

\begin{tabular}{|c|c|c|c|c|c|c|c|c|c|c|c|c|}
\hline \multirow[b]{3}{*}{ Total cases, $n(\%)$} & \multicolumn{12}{|c|}{ Patients atypical clinical manifestations } \\
\hline & \multicolumn{2}{|c|}{ Total cases } & \multicolumn{2}{|c|}{ Osteoarticular } & \multicolumn{2}{|c|}{ Hepatosplenic } & \multicolumn{2}{|c|}{ Lymphadenopathy } & \multicolumn{2}{|c|}{ Neuroretinitis } & \multicolumn{2}{|c|}{ Encephalitis } \\
\hline & \multicolumn{2}{|l|}{16} & 9 & (56) & 2 & $(13)$ & 2 & $(13)$ & 2 & (13) & 1 & (6) \\
\hline Age, mean \pm SD & \multicolumn{2}{|c|}{$8.0 \pm 4.5$} & \multicolumn{2}{|c|}{$6.4 \pm 4.9$} & \multicolumn{2}{|c|}{$11.9 \pm 3.8$} & \multirow{2}{*}{\multicolumn{2}{|c|}{$\begin{array}{l}9.4 \pm 3.5 \\
-\end{array}$}} & \multicolumn{2}{|c|}{$\begin{array}{l}11.0 \pm 1.4 \\
-\end{array}$} & \multicolumn{2}{|l|}{5.7} \\
\hline $\begin{array}{l}\text { Sex (M), } n(\%) \\
\text { Past history }\end{array}$ & 8 & $(50)$ & 5 & (56) & 2 & $(100)$ & & & - & & 1 & $(50)$ \\
\hline $\begin{array}{l}\text { Immunosupression, } n(\%) \\
\text { Epidemiological exposure }\end{array}$ & $=$ & & $=$ & & $=$ & & 二 & & 二 & & 二 & \\
\hline Cat exposure, $n(\%)$ & 14 & $(88)$ & 8 & (89) & 2 & $(100)$ & 1 & $(50)$ & 2 & $(100)$ & 1 & $(100)$ \\
\hline \multicolumn{13}{|l|}{ Season, $n(\%)$} \\
\hline $\begin{array}{l}\text { Spring } \\
\text { Summer }\end{array}$ & $\begin{array}{l}1 \\
7\end{array}$ & $\begin{array}{l}(19) \\
(50)\end{array}$ & $\begin{array}{l}1 \\
4\end{array}$ & $\begin{array}{l}(11) \\
(44)\end{array}$ & & & 2 & $(100)$ & 1 & $(100)$ & & \\
\hline Autumn & 8 & $(25)$ & 4 & (44) & 2 & $(100)$ & & & 1 & $(100)$ & 1 & (100) \\
\hline \multicolumn{13}{|l|}{ Time until $(\mathrm{D}) \dagger$, mean \pm SD } \\
\hline First evaluation & \multicolumn{2}{|c|}{$11.0 \pm 7.3$} & \multicolumn{2}{|c|}{$10.8 \pm 5.3$} & \multicolumn{2}{|c|}{$18.5 \pm 13.4$} & \multicolumn{2}{|c|}{$13.5 \pm 6.4$} & 1.0 & & \multicolumn{2}{|l|}{3.0} \\
\hline Clinical diagnosis & \multicolumn{2}{|c|}{$44.0 \pm 14.7$} & \multicolumn{2}{|c|}{$46.0 \pm 15.5$} & \multicolumn{2}{|c|}{$52.5 \pm 2.1$} & $41.0 \pm 8$ & & 15.0 & & 44.0 & \\
\hline Clinical signs and symptoms, $n(\%)$ & & & & & & & & & & & & \\
\hline Fever & 13 & (82) & 8 & (89) & 2 & $(100)$ & - & 2 & $(100)$ & 1 & $(100)$ & \\
\hline Pain stigma & 12 & (75) & 9 & $(100)$ & 1 & $(50)$ & - & 1 & $(50)$ & 1 & $(100)$ & \\
\hline Vision loss & 2 & (13) & - & - & - & 2 & (100) & - & & & & \\
\hline Seizures & 1 & (6) & - & - & - & - & 1 & $(100)$ & & & & \\
\hline $\begin{array}{l}\text { Constitutional symptoms } \\
\text { Signs on physical examination, } n(\%)\end{array}$ & 7 & $(44)$ & 3 & (33) & 2 & $(100)$ & - & 1 & $(50)$ & 1 & (100) & \\
\hline Lymphadenopathy & 11 & (69) & 6 & (67) & 2 & $(100)$ & 2 & $(100)$ & 1 & (50) & - & \\
\hline Hepatosplenomegaly & 1 & (6) & 1 & (11) & - & & - & & - & & - & \\
\hline Rash & 2 & (13) & 2 & (22) & - & & - & & - & & - & \\
\hline Meningeal signs & 2 & (13) & 1 & (11) & - & & - & & - & & 1 & (100) \\
\hline $\begin{array}{l}\text { Torticollis } \\
\text { US/CT scan findings, } n(\%)\end{array}$ & 1 & (6) & 1 & (11) & - & & - & & - & & - & \\
\hline $\begin{array}{l}\text { Hepatosplenic nodules } \\
\text { Laboratory diagnosis, } n \text { (\%) }\end{array}$ & 4 & $(25)$ & 2 & (22) & 2 & $(100)$ & - & & - & & - & \\
\hline Seroconversion (IFA) & $16 / 16$ & $(100)$ & 9 & $(100)$ & 2 & $(100)$ & 2 & (100) & 2 & $(100)$ & 1 & (100) \\
\hline Positive PCR & $2 / 8$ & $(25)$ & 1 & (11) & 1 & $(50)$ & - & & - & & - & \\
\hline $\begin{array}{l}\text { HPE with granulomatous inflammation } \\
\text { Treatment, } n(\%)\end{array}$ & $2 / 4$ & $(50)$ & 1 & (11) & 1 & (50) & - & & - & & - & \\
\hline Azithromycin & 3 & (19) & 1 & (11) & - & & 2 & $(100)$ & - & & - & \\
\hline Rifampicin & 1 & (6) & 1 & (11) & - & & - & & - & & - & \\
\hline Rifampicin + Azithromycin & 2 & $(13)$ & 2 & (22) & - & & - & & - & & - & \\
\hline Rifampicin + Ciprofloxacin & 5 & (31) & 3 & (33) & 2 & $(100)$ & - & & - & & - & \\
\hline Rifampicin + Doxycyclin & 3 & (19) & 1 & (11) & - & & - & & 2 & $(100)$ & - & \\
\hline Ceftriaxone & 1 & (6) & - & & - & & - & & - & & 1 & (100) \\
\hline Cotrimoxazole & 1 & (6) & 1 & (11) & - & & - & & - & & - & \\
\hline Full recovery, $n(\%)$ & 16 & $(100)$ & 9 & $(100)$ & 2 & (100) & 2 & $(100)$ & 2 & (100) & 2 & (100) \\
\hline
\end{tabular}

†One missing value for time until 1st evaluation and diagnosis. CT, computed tomography; D, days; IFA, immunofluorescence assay; M, male; PCR, polymerase chain reaction; SD, standard deviation; US, ultrasound.

performed in $25 \%$ of the patients, requiring alternative diagnosis exclusion like tuberculosis or malignancy.

In our series, regional lymphadenopathy was not the disease's hallmark, present in only $69 \%$ of cases. The main lymph node involvement region was however associated with the nodes that drained the area where cat scratches usually occur, such as the axilla, the neck, and the groin. ${ }^{9}$ Symptoms such as fever, malaise, anorexia, weight loss, headache, rash or arthralgia, as well as, fever of unknown origin and multiple microabscesses in liver and/or spleen were more common in systemic involvement and few cases presented with the typical CSD manifestations.

In our study, both cases of hepatosplenic disease presented with prolonged fever, lymphadenopathy and hepatosplenic microabscesses, as well as verified in the two other patients with 
hepatosplenitis overlapping osteomyelitis. Imaging exams were necessary to confirm the organomegalies. As for the therapeutic approach, even though no controlled trials of antibiotic therapy were performed, rifampicin has been recommended in combination with gentamicin, trimethoprim-sulfamethoxazole, fluoroquinolones or macrolides. In our series, clinical improvement was verified with the association of rifampicin and ciprofloxacin for 6 weeks.

As for the ophthalmological manifestations, although Parinaud's oculoglandular syndrome has been the most frequent encountered $(4-6.1 \%),{ }^{11,13}$ we had no reported cases, possibly due to its benign evolution and the unusual hospital admission criteria. Neuroretinitis was the only ophthalmological atypical manifestation in our series. Vision loss at presentation warranted the use of steroids in both patients which may have been helpful in their subsequent recovery. Doxycycline and rifampicin were also used in combination for $2-4$ weeks, ${ }^{14,15}$ as it has been recommended in paediatric age.

Among neurological manifestations, encephalopathy has been described as the most frequent in $0.25-2.4 \%$ of patients. ${ }^{11,13}$ In this study, epidemiologic context with several farm animals, besides cats and dogs, made the exclusion of other zoonosis essential and delayed the diagnosis and directed therapy. Although to this day, there are no guidelines for the treatment of these atypical manifestation, for the neurological presentations, the association of doxycycline and rifampicin have been useful.

As for osteomyelitis, despite being known as a rare manifestation of atypical bartonellosis, it was the most common presentation in our study, accounting for more than half of our cases. As a reference hospital for paediatric orthopaedics, it is comprehensible that this atypical manifestation prevalence is high. RoubaudBaudron et al. ${ }^{16}$ reviewed 41 children and 11 adults, with osteoarticular bartonellosis, the majority of which with unifocal bone involvement $(72 \%)$. Most cases presented as fever and pain $(85 \%)$; the main affected locations were the vertebrae, the pelvis, and the rib cage. In our series, unifocal involvement was similar $(89 \%)$ and while the vertebrae were affected in $55 \%$ of the cases, the sacroiliac joint was also involved in a considerable number of cases $(33 \%)$. Clavicle and tibiotarsi joint involvement was more rare. Overlapping hepatosplenitis occurred in $22 \%$ of cases, similarly to the reported in their review $(26 \%)$, pointing out that physicians should consider bartonellosis in children who have osteomyelitis or new onset hepatosplenic abnormalities. The apparent higher sensitivity of PCR in lymph node or abscess specimen, rather than blood samples, was corroborated by our study, in which all blood PCR for Bartonella spp. were negative and one lymph node PCR was positive. ${ }^{16}$ The timing of the exam is crucial in these cases, as negative results have been described in the initial phases, latter evolving for a positive result. As for the therapeutic approach, in spite of the controversy on the choice of the best medicine, to this day, most authors praise the importance of antibiotics with good bone penetration and molecules as cotrimoxazole, ciprofloxacin and rifampicin, as well as, doxycycline and azithromycin have been recomended. ${ }^{16}$

We can, therefore, understand that, concerning the different atypical manifestations of bartonellosis, numerous antibiotic combinations have been used, lacking evidence about their effectiveness and the current practice is based on case reports, small series and personal experience. Commonly used antibiotics, such as erythromycin, doxycycline and antimycobacterial drugs, have been pointed as adequate for these patients, ${ }^{11}$ but the final choice should always rely on the clinical manifestations.

Immunodeficiencies were excluded in a significant number of children that remain healthy to this day. Even though atypical manifestations of CSD have been known to occur mainly in immunocompromised patients, recent studies have documented that some of the manifestations are also prevalent in the immunocompetent ones. ${ }^{16}$

This study has some limitations in terms of representativeness of cases. However, because these are cases with atypical manifestations, they are not very frequent. It should also be noted that the high frequency of atypical presentations of bartonellosis in our hospital is most likely due to selection bias of reviewing cases that required admission to a tertiary paediatric hospital.

\section{Conclusions}

Even though regional lymphadenopathy is considered the hallmark of CSD, in few cases, this disease can present with atypical manifestations and a high index of clinical suspicion and the epidemiological context is mandatory. The reduced number of cases with atypical bartonellosis makes it difficult to make correlations between the clinical presentations, possible risk factors and best treatment. Multicentric studies to better understand this entity are necessary as, to this day, it remains a challenge to each physician.

\section{Acknowledgements}

The authors would like to thank the laboratory support for serological assays to Teresa Luz, Paulo Parreira, Ana Sofia Santos and Maria Salomé Gomes (Centre of Study of Vectors and Infectious Diseases, National Institute of Health Dr. Ricardo Jorge, Lisbon, Portugal).

\section{References}

1 Staggemeier R, Venker CA, Klein DH, Petry M, Spilki FR, Cantarelli VV. Prevalence of Bartonella henselae and Bartonella clarridgeiae in cats in the south of Brazil: A molecular study. Mem. Inst. Oswaldo Cruz 2010; 105: 873-8.

2 Da Silva K, Chussid S. Cat-scratch disease: Clinical considerations for pediatric dentist. Pediatr. Dent. 2009; 31: 58-62.

3 Chen TC, Lin WR, Lu PL, Lin CY, Chen YH. Cat-scratch disease from a domestic dog. J. Formos. Med. Assoc. 2007; 106: S65-8.

4 Ulug Mehmet. Evaluation of cat-scratch disease cases reported from Turkey between 1996 and 2013 and review of the literature. Cent. Eur. J. Public Health 2015; 23: 170-5.

5 Sanguinetti-Morelli D, Angelakis E, Richet H, Davoust B, Rolain JM, Raoult D. Seasonality of cat-scratch disease, France, 1999-2009. Emerg. Infect. Dis. 2011; 17: 705-7.

6 English R. Cat-scratch disease. Pediatr. Rev. 2006; 27: 123-8.

7 Arisoy ES, Correa AG, Wagner ML, Kaplan SL. Hepatosplenic catscratch disease in children: Selected clinical features and treatment. Clin. Infect. Dis. 1999; 28: 778-84.

8 Habot-Wilner Z, Trivizki O, Goldstein M et al. Cat-scratch disease: Ocular manifestations and treatment outcome. Acta Ophthalmol. 2018; 96: e524-e32. 
9 Florin TA, Zaoutis TE, Zaoutis LB. Beyond cat scratch disease: Widening spectrum of Bartonella henselae infection. Pediatrics 2008; 121 e1413-25.

10 Bass JW, Freitas BC, Freitas AD et al. Prospective randomized double-blind placebo-controlled evaluation of azithromycin for treatment of cat-scratch disease. Pediatr. Infect. Dis. J. 1998; 17 447-52.

11 Margileth A. Recent advances in diagnosis and treatment of catscratch disease. Curr. Infect. Dis. Rep. 2000; 2: 141-6.

12 Zeaiter Z, Fournier PE, Greub G, Raoult D. Diagnosis of Bartonella endocarditis by real-time nested PCR assay using serum. J. Clin. Microbiol. 2003; 41: 919-25.
13 Carithers HA. Cat-scratch disease: An overview based on a study of 1200 patients. Am. J. Dis. Child. 1985; 139: 1124-33.

14 Rolain JM, Brouqui P, Koehler JE, Manguina C, Dolan MJ, Raoult D. Recommendations for treatment of human infections caused by Bartonella species. Antimicrob. Agents Chemother. 2004; 48: 1921-33.

15 Kalogeropoulos D, Asproudis I, Stefanioutou M et al. Bartonella henselae- and quintana-associated uveitis: $A$ case series and approach of a potentially severe disease with a broad spectrum of ocular manifestations. Int. Ophtalmol. 2019; 39: 2505-15.

16 Roubaud-Baudron C, Fortineau N, Goujard C, Le Bras P, Lambotte O. Maladie des griffes du chat avec localisations osseuses: Une observation et revue de la literature. Rev. Méd. Interne 2009; 30: 602-8.

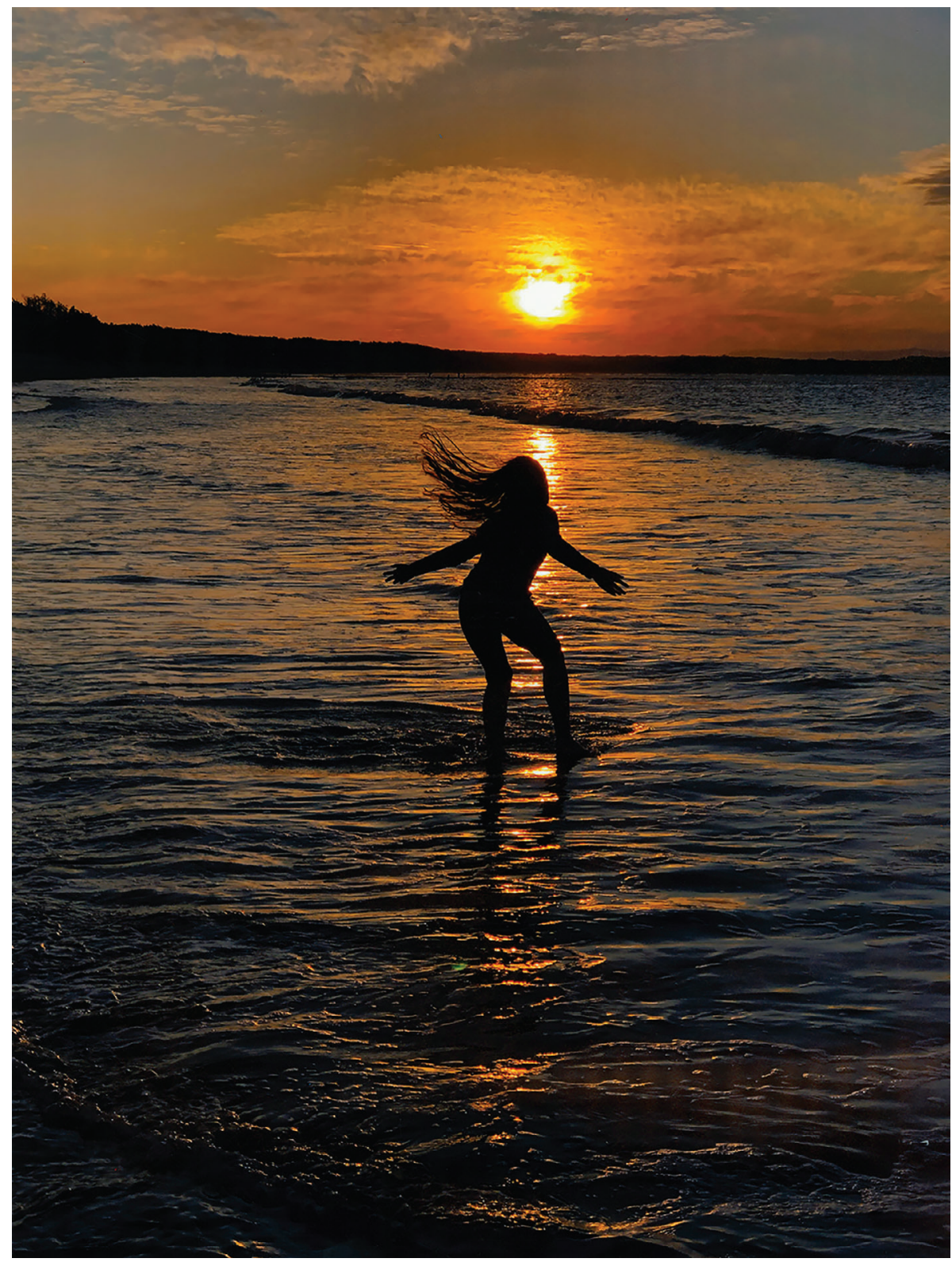

Sunset at Currarong by Olive Coulthard-Coves (11) from Operation Art 2020 